\title{
DEL HUERTO A LA CIUDAD: AGRICULTURA FAMILIAR Y APROVISIONAMIENTO URBANO EN LA SIERRA ECUATORIANA
}

\author{
Nasser Rebaï \\ UMR PRODIG 8586. Universidad Paris I Panthéon-Sorbonne, \\ nass.reb@hotmail.fr
}

\section{RESUMEN}

En Ecuador, la migración campesina es el motor principal de las recomposiciones del medio rural andino. En la provincia del Azuay, la disminución de la mano de obra en los últimos años ha provocado una desestructuración de las familias campesinas y una reorganización lógica de las tareas agrícolas. Sin embargo, gracias a la cercanía de la ciudad de Cuenca, los campesinos desarrollan una actividad comercial intensa, orientada hacia la ganadería y la agro-ecología. Si por una parte podemos considerar esta dinámica como el símbolo del renacimiento de la agricultura familiar, por otra parte falta todavía una visión ambiciosa para llegar verdaderamente a un modelo de desarrollo rural sustentable que erradique la pobreza rural y garantice la soberanía alimentaria en esta región andina.

Palabras clave: Ecuador, Andes, agricultura familiar, aprovisionamiento urbano.

\section{ABSTRACT}

In Ecuador, the peasant migration is the main engine of the Andean rural recompositions. In the province of Azuay, the decrease in the labour force in recent years has caused a destructuring of rural families and a logical reorganization of the agricultural tasks. However, thanks to the proximity of the city of Cuenca, farmers develop an intense commercial activity, oriented towards livestock and the Agroecology. If on the one hand we can consider this dynamic as the symbol of the Renaissance of family farming, on the other hand lack still an ambitious vision to truly reach a model of sustainable rural development that will eradicate rural poverty and ensure food sovereignty in the Andean region.

Key words: Ecuador, Andes, farming, urban supply. 


\section{INTRODUCCIÓN}

En los últimos años, la migración ha sido un factor de cambio fundamental de las prácticas campesinas en la región andina del Ecuador. La disminución progresiva de la mano de obra ha dado lugar a una transformación del uso del suelo, que implica una mutación de la actividad agropecuaria y de la economía familiar. A través de este artículo nos proponemos hacer un análisis más amplio, observando precisamente la redefinición de las relaciones campo-ciudad en la provincia del Azuay, el corazón histórico de la migración ecuatoriana. Por eso nos vamos a focalizar en el caso de la parroquia Octavio Cordero Palacios, y organizaremos nuestra reflexión a partir de una pregunta simple: ¿en qué medida el contexto migratorio ha favorecido la emergencia de redes de productores que participan activamente en el aprovisionamiento agrícola de la ciudad de Cuenca?

\section{PROPONER UN PUNTO DE VISTA ORIGINAL SOBRE LAS MUTACIONES ACTUALES DEL MEDIO RURAL ANDINO}

En Ecuador, la migración es un fenómeno de importancia nacional: en 2008, entre 1.4 y 1.6 millones de ecuatorianos vivían en el extranjero (FLACSO, 2008: 15). En los últimos años, las publicaciones sobre el tema migratorio han sido numerosas y han permitido debatir sobre cuestiones tanto sociales (Herrera et al., 2006, 2008) como económicas (Acosta et al., 2006; Nieto, 2005). Nosotros nos proponemos dar una visión geográfica de los efectos de la migración, analizando precisamente las recomposiciones agrarias en la región andina. Por eso elegimos una localidad con una historia y una situación geográfica singulares, como ejemplo de las mutaciones del medio rural en el contexto migratorio actual.

UNA MIGRACIÓN ANTIGUA EN UNA LOCALIDAD DE LA PERIFERIA DE CUENCA

En primer lugar, realizamos un trabajo de investigación en la parroquia Juncal, ubicada en la provincia del Cañar, donde pudimos constatar que la migración continua de la población campesina desde hace doce años influye sobre el uso del suelo. Observamos que, debido a la falta 
de mano de obra, las familias reducen cada vez más las superficies cultivadas y prefieren dedicarse a la ganadería, para tener ingresos regulares con la venta de leche (Rebaï, 2008).

Luego, para seguir nuestro análisis de los efectos de la migración en la sierra ecuatoriana, y para no limitarnos a un estudio de caso, tuvimos que orientarnos hacia una localidad cuya historia migratoria era más antigua, para describir sus efectos concretos en una escala temporal más amplia. Lógicamente, nos dirigimos hacia la provincia del Azuay, en donde la migración internacional de la población campesina empezó en los años cincuenta (Carpio, 1992).

Sin embargo, al cambiar de marco regional, tuvimos que integrar en nuestra reflexión el tema de la influencia urbana sobre la agricultura campesina. Más allá del contexto migratorio, tuvimos que considerar el crecimiento demográfico de la ciudad Cuenca, la cabecera provincial del Azuay, la cual mantiene relaciones intensas con las localidades rurales de su periferia desde su fundación en el siglo XVI (Poloni-Simard, 2006). Al profundizar en la dinámica poblacional, se reporta que la población de la tercera ciudad ecuatoriana pasó de 87,415 a 277,374 habitantes entre 1974 y 2000 (Instituto Nacional de Estadísticas y Censos, INEC), lo que probablemente tuvo consecuencias importantes sobre la actividad agropecuaria provincial en los últimos años.

Así, nos dirigimos hacia la parroquia Octavio Cordero Palacios ubicada a $22 \mathrm{~km}$ de la ciudad de Cuenca. Respecto a nuestra primera experiencia de investigación en la provincia del Cañar, la cercanía de un polo urbano nos pareció un elemento nuevo muy interesante, ya que podíamos estudiar la evolución de la agricultura familiar en un contexto a priori favorable. En Juncal, la migración sigue siendo la única alternativa económica porque esta localidad pertenece a un «subespacio de la periferia pasiva [del territorio nacional]» (Deler, 2007: 375), donde el desarrollo de la agricultura comercial es casi imposible, debido al alejamiento de la grandes ciudades del país. Por el contrario, la proximidad urbana puede ser una ventaja para el sostenimiento de la agricultura familiar al favorecer la emergencia de redes cortas de aprovisionamiento, como lo han mostrado geógrafos franceses en diferentes estudios de caso africanos (Chaléard, 1996; Tallet, 1999; Calas, 1999). Antes de realizar nuestro trabajo de campo, 
tuvimos que constituir una base de informaciones para caracterizar nuestra nueva zona de estudio.

Figura 1. Mapa de ubicación de la parroquia Octavio Cordero Palacios

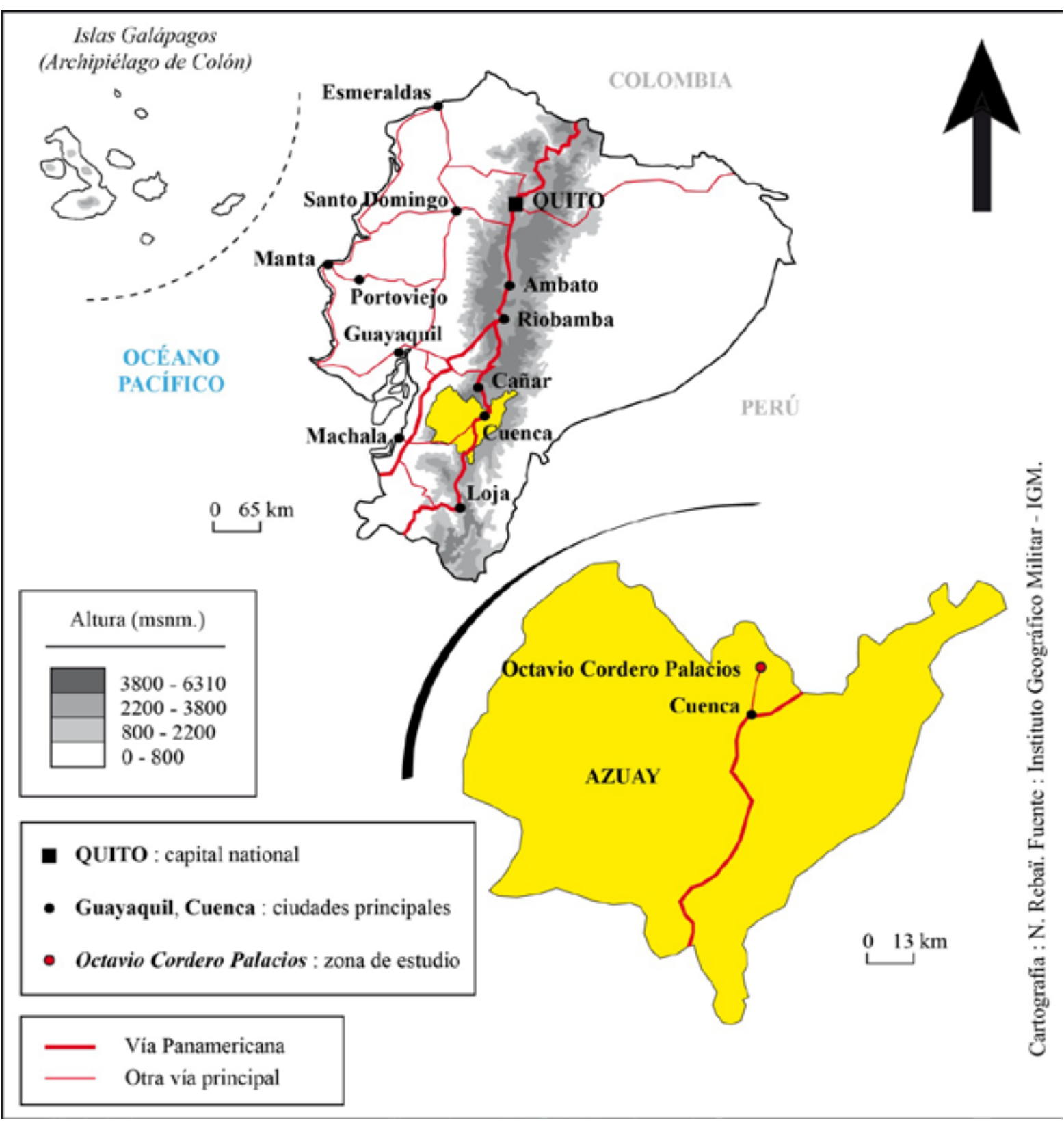

Fuente: Instituto Geográfico Militar. 
DISMINUCIÓN POBLACIONAL Y CAMBIOS AGRARIOS EN LA PARROQUIA OCTAVIO CORDERO PALACIOS

Durante la primera etapa de nuestro trabajo de investigación, en el campo, consultamos los datos del INEC y los del Instituto de Estudios de Régimen Seccional del Ecuador (IERSE, 2003). Eso nos permitió constatar dos fenómenos clave: una caída poblacional de $33.4 \%$ en la parroquia Octavio Cordero Palacios, entre 1982 y 2001, y un cambio profundo en el uso del suelo caracterizado por una disminución de las superficies cultivadas (-9\%) y un aumento excepcional de las áreas de pasto (+84\%), entre 1991 y 2001.

Cuadro 1. Evolución poblacional en la parroquia Octavio Cordero Palacios (1982-2001).

\begin{tabular}{|c|c|c|c|}
\hline Año & 1982 & 1990 & 2001 \\
\hline Número de habitantes & 3134 & 2767 & 2178 \\
\hline
\end{tabular}

Fuente: INEC.

Cuadro 2. Evolución del uso del suelo en la parroquia Octavio Cordero Palacios (1991-2007).

\begin{tabular}{|c|c|c|c|}
\hline \multirow{2}{*}{ Uso del suelo } & \multicolumn{2}{|c|}{$\begin{array}{c}\text { Superficies } \\
\text { en hectáreas }\end{array}$} & \multirow{2}{*}{$\begin{array}{c}\text { Evolución } \\
\text { en \% }\end{array}$} \\
\cline { 2 - 3 } & 1991 & 2001 & \\
\hline Cultivos & 1194,2 & 1088 & $-8,9$ \\
\hline Bosques & 596,1 & 479,6 & $-19,5$ \\
\hline Páramos & 173,2 & 152,9 & $-11,7$ \\
\hline Pasto & 300,9 & 554,4 & $+84,2$ \\
\hline
\end{tabular}

Fuente: IERSE (2003).

Con esta información podíamos considerar legítimamente que la disminución de la mano de obra era el factor principal del cambio en el uso del suelo, y suponer al mismo tiempo que las transformaciones podían ser mucho más importantes entre 2001 y 2012, considerando el aumento de la migración tanto en esta zona como en el resto del país. ${ }^{1}$ Para verificar esta hipótesis entramos en la dimensión práctica de nuestra investigación al vincularnos con la población local. Así, consultamos a cincuenta personas y nos dedicamos a un trabajo de entrevistas según dos modos: 
- Primero, queríamos saber cómo era la vida campesina antes de la migración. Para ello realizamos 16 entrevistas con ancianos y tenientes políticos (representantes de la junta parroquial y de las comunidades de la localidad), para enterarnos de las mutaciones del espacio agrario local durante la segunda mitad del siglo XX; les preguntamos cuáles eran las diferencias principales entre la agricultura de ayer y la de hoy.

- Luego, queríamos entender cómo evolucionaron las prácticas campesinas a lo largo de las últimas décadas. Por eso, hicimos entrevistas con 38 agricultores, para conocer la organización de las tareas cotidianas en cada finca. Concretamente, consultamos tanto familias con migrantes (28) como sin migrantes (10), para poder enfatizar la diversidad de las explotaciones en la localidad, a pesar de la dificultad para encontrar las familias sin ningún vínculo con la migración.

Este trabajo, en cada hogar, nos permitió precisar los efectos de la migración sobre las estructuras familiares campesinas, que sobrepasaba las estadísticas demográficas oficiales del INEC. Así, constatamos que desde 1976 el 29\% de la mano de obra global había salido a los Estados Unidos de Norteamérica, y que solamente se quedó el 29\% de las personas para dedicarse a la agricultura a tiempo completo. Por lo demás, el 17\% de la gente tiene un empleo urbano y, por consecuencia, no puede trabajar la tierra regularmente; mientras que el 25\% de los individuos constan de niños y de ancianos que no ayudan en la finca. En este contexto, se produjo lógicamente una recomposición progresiva de las tareas agrícolas en los últimos años, y para entenderlas nos interesamos principalmente en las familias con migrantes, para saber cómo siguen trabajando la tierra a pesar de la falta de mano de obra.

\section{ENTRE CAMPO Y CIUDAD: LAS DOS CARAS DE LA INVESTIGACIÓN}

Tras consultar a las familias con migrantes sobre sus lógicas de trabajo obtuvimos respuestas muy claras. Para ellas, el objetivo es vincularse con el mercado urbano de Cuenca para sacar ingresos regulares. En consecuencia, las personas que se quedan, las mujeres en particular, 
prefieren aumentar por un lado las superficies de pasto para vender leche y criar cuyes, y por otro lado intensificar el trabajo sobre micro huertos para vender hortalizas. Así, desaparecen progresivamente el maíz, el haba, el frijol y los tubérculos. Asistimos, pues, a un cambio profundo en las prácticas agrícolas: las superficies de cultivos de ciclo largo se reducen cada vez más porque no aseguran la supervivencia diaria de los hogares.

Fotografía 1. El nuevo paisaje agrario en la parroquia Octavio Cordero Palacios. Los tres componentes del paisaje agrario en la parroquia Octavio Cordero Palacios: el huerto, el ganado y la casa nueva, símbolo del éxito migratorio.

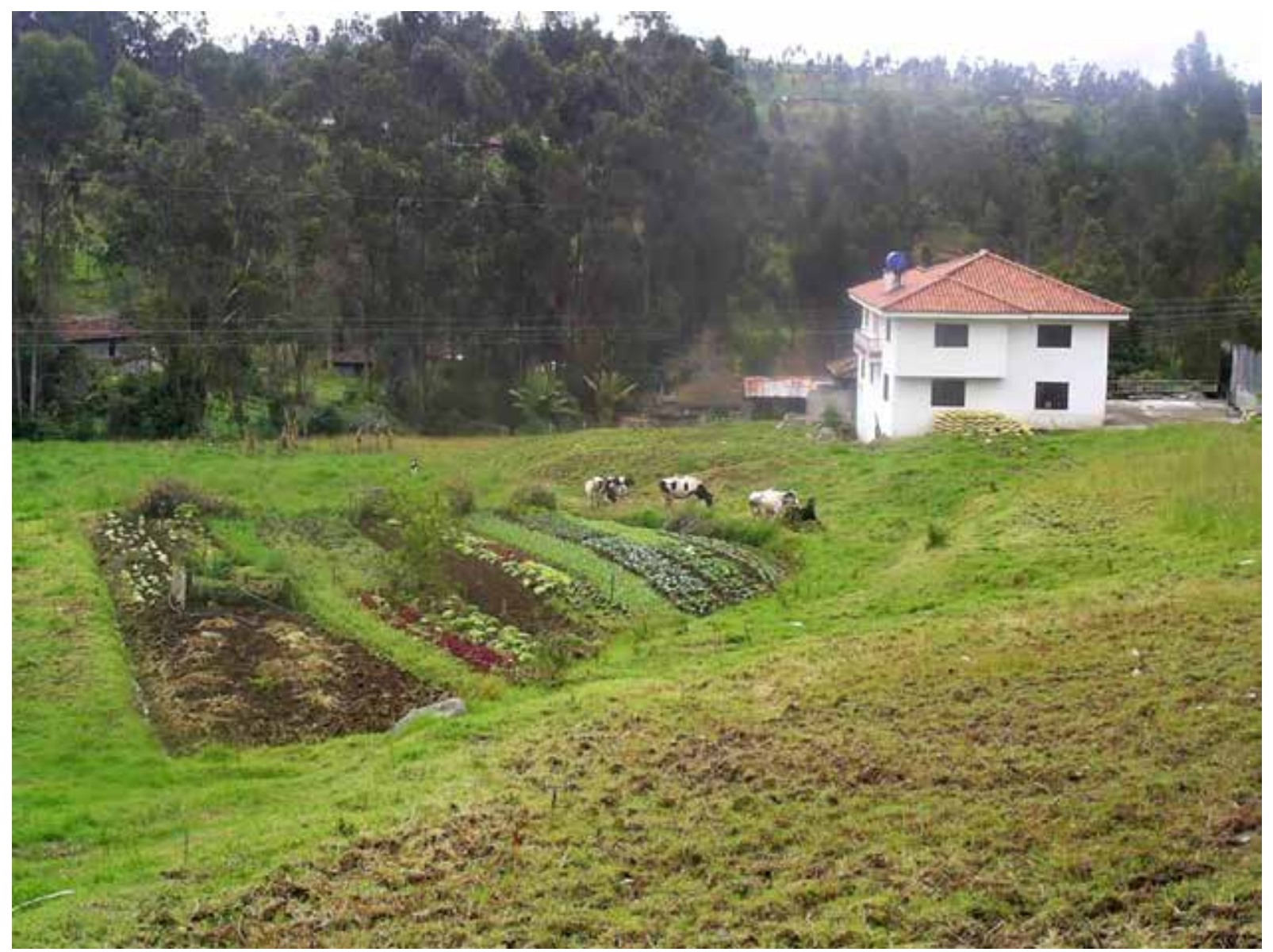

Fuente: Rebaï (2009).

Así, después de una mutación profunda en el uso del suelo, nos enteramos que la migración había provocado la redefinición de las relaciones campo-ciudad. Si hasta finales de los años noventa las 
familias de la parroquia Octavio Cordero Palacios se iban a Cuenca para comprar una parte de sus necesidades alimentarias, ahora constatamos que participan activamente en el aprovisionamiento urbano, al vender hortalizas, frutales, productos lácteos y pequeños animales. No obstante, no podíamos limitarnos a esta constatación y teníamos que orientar nuestra metodología para entender las lógicas de inserción urbana de los campesinos. Por eso realizamos otra serie de entrevistas y de encuestas. Eso constituyó la segunda etapa de nuestra investigación, en la ciudad.

- Primero, hicimos seis entrevistas con técnicos del Programa de Agricultura Urbana (PAU) del municipio de Cuenca, del Centro de Reconversión Económico del Austro $^{2}$ (CREA) y del Centro de Desarrollo y de Investigación Rural (CEDIR), cuya meta común es la promoción de la agricultura comercial y agro-ecológica en la provincia de Azuay.

- Luego, realizamos el seguimiento comercial de cinco familias que trabajan en grupo, entre septiembre de 2008 y mayo de 2009 (9 meses). Esta encuesta económica global fue posible gracias a la existencia de un libro de cuentas que pudimos consultar regularmente, con el acuerdo de los campesinos y ayudándoles a mejorar la contabilidad.

- Por último, consultamos a los administradores de cinco mercados cuencanos para debatir sobre el rol de los campesinos locales en el aprovisionamiento urbano.

En definitiva, pudimos estudiar la recomposición de la actividad campesina como si fuera una cadena: al inicio nos dedicamos a la parte agraria y a los efectos de la migración sobre el uso del suelo, y luego estudiamos la dimensión comercial y la complementariedad creciente entre lo rural y lo urbano. Ahora vamos a desarrollar este último tema.

\section{INSERCIÓN URBANA Y AGRICULTURA COMERCIAL EN LA PROVINCIA DEL AZUAY}

Hasta finales de la década de los noventa, los campesinos de la parroquia Octavio Cordero Palacios no vendían productos agrícolas, o lo hacían de manera muy excepcional en algunas 
ferias rurales, o a veces como vendedores informales en las calles de Cuenca. Las fuentes monetarias para los gastos agrícolas y domésticos provenían principalmente de las actividades extra-agrícolas (artesanía, migraciones temporales a la costa para trabajar en las plantaciones bananeras o cacaoteras y migración internacional). Pero desde hace poco la emergencia de redes regionales de productores ha provocado un cambio importante en la economía familiar.

\section{APOYOS INSTITUCIONALES Y FORMACIÓN DE REDES DE PRODUCTORES}

Hace quince años, los campesinos de la zona empezaron a vincularse a los mercadosgracias a la intervención del cura de la parroquia, quien los animó a producir frutas y hortalizas para ya no depender de las remesas. En la provincia existía una sinergia entre la Iglesia y las ONG desde hacía más de quince años, que correspondía a una voluntad común de viabilizar la condición campesina después de la Reforma Agraria (1964/1974), creando grupos de trabajo y de producción en varias localidades del Azuay. En los años noventa aparecieron en el debate sobre el desarrollo rural regional algunas instituciones como la Universidad de Cuenca o el Centro de Agricultura Biológica, para investigar en el campo y encontrar alternativas a la pobreza rural y a la migración. En los inicios del 2000, el municipio de Cuenca y el CREA actuaron de manera concreta al crear dos asociaciones de productores agro-ecológicos, que reunían en 2009 más de 300 familias.

Según los diferentes técnicos que trabajan sobre el tema, la agro-ecología constituye un enfoque muy concreto del desarrollo rural, justamente porque garantiza el acceso de los campesinos al mercado, atribuyéndoles títulos de productores limpios (que trabajan sin químicos). Desde un punto de vista puramente comercial, eso permitió tener desde el inicio un ambiente de confianza con los consumidores, quienes ahora prefieren comprar los productos locales y favorecer la agricultura azuaya. La formación de dos redes regionales de pequeños productores constituyó también un marco institucional muy importante para la inserción urbana y para luchar (oficiosamente) contra la informalidad. Así, entre el campo y la ciudad se desarrollaron nuevas formas de complementariedad que modificaron tanto los espacios urbanos de venta, como la economía campesina. 
Fotografías 3, 4, 5 y 6. La visibilidad de las asociaciones campesinas en los mercados cuencanos.

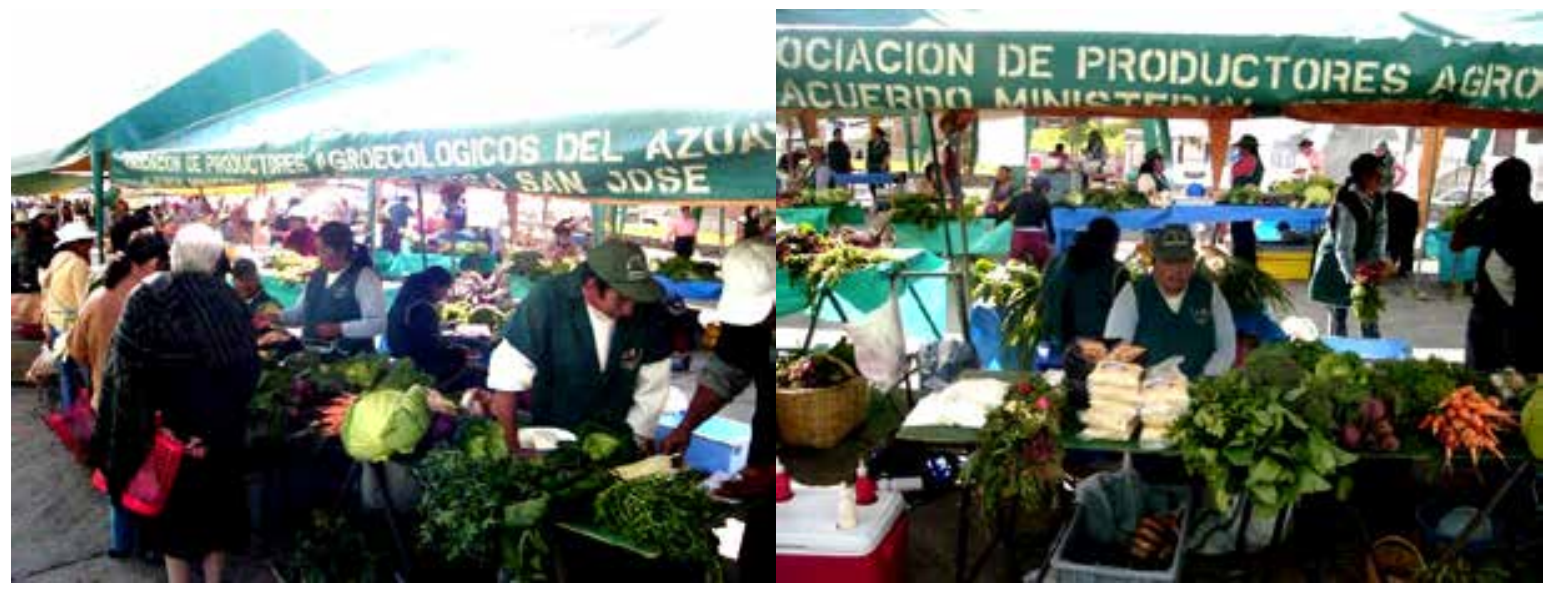

Las carpas y los uniformes verdes permiten una mejor visibilidad de los Productores Agroecológicos del Azuay (PAA-Municipio de Cuenca) en la feria de Miraflores...
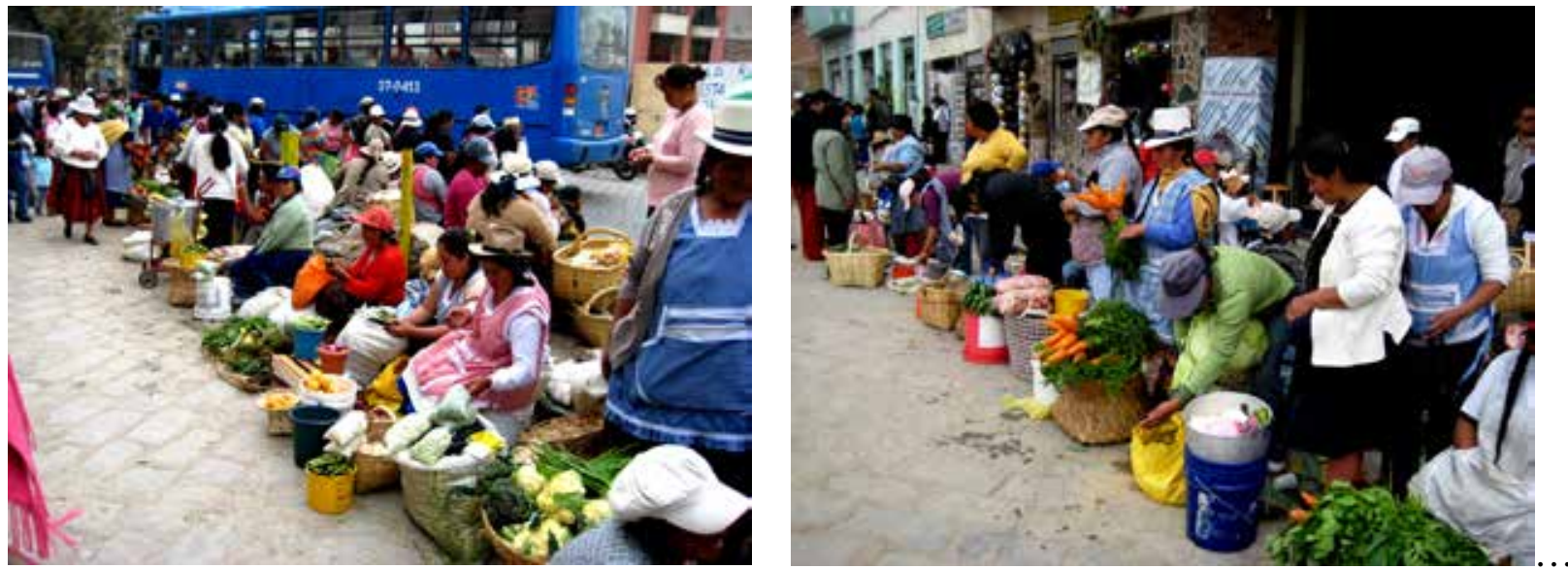

mientras que las vendedoras informales del mercado de la 9 de Octubre luchan entre los autobuses y, a veces, contra la policía.

Fuente: Rebaï (2009).

TRABAJOS COLECTIVOS Y CAMBIOS EN LA ECONOMÍA FAMILIAR

En la parroquia Octavio Cordero Palacios existen varios grupos de trabajo que nacieron al final de los noventa para luchar contra la falta de mano de obra. Entre ellos, el grupo de productores agro-ecológicos Bajo Invernadero ${ }^{3}$ es uno de los más dinámicos, reúne cinco familias y recibe el apoyo técnico del CREA desde 2004. Dentro del grupo, diez personas se dedican diariamente a 
la agricultura para mantener a 24; mientras que desde 1995 la mano de obra global se redujo en un 30\% por la migración. Las salidas al mercado se desarrollan dos veces a la semana, lo que permite a las cinco familias tener ingresos muy regulares. Entre las tareas agrícolas y la parte comercial, los miembros del grupo Bajo Invernadero trabajan colectivamente haciendo mingas para el trabajo de la tierra y actuando como un equipo de venta los días de mercado.

Fotografías 7 y 8 . Vendedores en el mercado del CREA. Productores del grupo Bajo Invernadero vendiendo hortalizas.

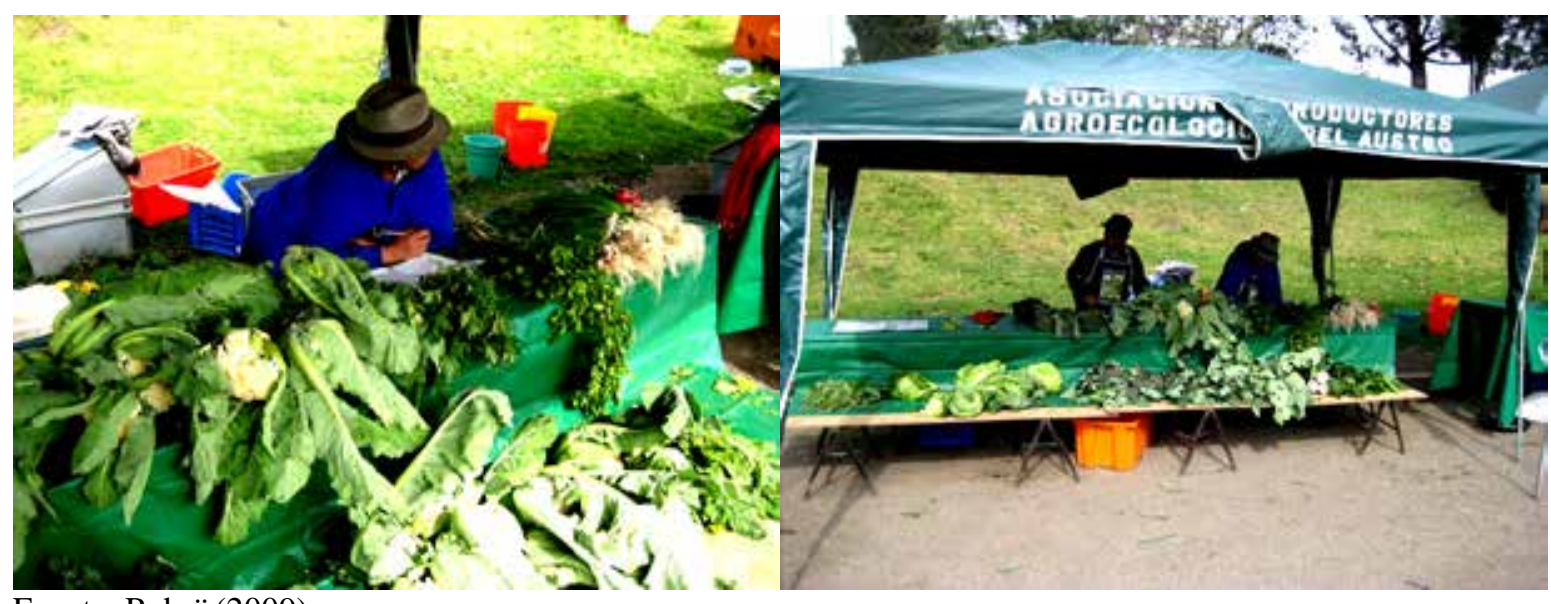

Fuente: Rebaï (2009).

Según las entrevistas que tuvimos con los diferentes miembros del grupo, antes de pertenecer a la Asociación de Productores Agro-ecológicos del Austro, ninguno de ellos se iba a Cuenca para vender cultivos. Ahora, todos tienen ingresos regulares, como lo indica el cuadro siguiente: 
Cuadro 3. Detalle de los ingresos agrícolas (en dólares) de cinco productores agro-ecológicos de la parroquia Octavio Cordero Palacios (septiembre de 2008 - mayo de 2009).

\begin{tabular}{|c|c|c|c|c||c||c|}
\hline Productor & $\begin{array}{c}\text { Hortalizas } \\
\text { y frutales }\end{array}$ & $\begin{array}{c}\text { Pollos y } \\
\text { huevos }\end{array}$ & Cuyes & Lácteos & Total & $\begin{array}{c}\text { Ingreso } \\
\text { promedio } \\
\text { mensual }\end{array}$ \\
\hline 1 & 1179,4 & 54,9 & 81,5 & 716,6 & 2032,4 & 225,8 \\
\hline 2 & 485,45 & 280,1 & 100 & 742,35 & 1607,9 & 178,6 \\
\hline 3 & 248,1 & 104,9 & 196,8 & 94 & 643,8 & 71,5 \\
\hline 4 & 280,25 & 64,75 & 66 & 248,7 & 659,7 & 73,3 \\
\hline 5 & 519,9 & 0 & 10 & 159,25 & 682,15 & 75,8 \\
\hline
\end{tabular}

Fuente: Libro de cuentas, grupo Bajo Invernadero. Cálculos: Rebaï.

A pesar de los costos de transporte (4 dólares semanales/productor), de los impuestos en los mercados (5 dólares mensuales/productor) y de las compras regulares de abono orgánico (entre 5 y 10 dólares mensuales/finca), la actividad agropecuaria se ha vuelto una fuente monetaria importante, con un ingreso promedio de 125 dólares mensuales por cada productor.

Sin embargo, si consideramos las ventas directas en las fincas, en particular la de leche a los intermediarios, los ingresos pueden alcanzar más de 400 dólares mensuales (productor 1). Si nos interesamos por las ventas informales de pequeños animales (directamente en las fincas o en las calles cuencanas), igualmente constatamos un aumento de los ingresos agropecuarios de $50 \%$ (productor 2) y de 80\% (productor 3). Entonces, podemos decir que existe una fuerte incremento de la producción pecuaria con fines comerciales; no obstante, las ventas de hortalizas y frutas constituyen un fuerte porcentaje de los ingresos agropecuarios, (42\% para el productor 4 y 76 \% para el productor 5) .

Después de la descripción de la importancia de la actividad pecuaria, se necesita dar otra precisión sobre el rol fundamental de las fuentes monetarias exteriores para la economía familiar. Actualmente, los empleos locales y las remesas siguen constituyendo más del 50\% de los ingresos globales de este grupo. Solamente una explotación vive integralmente de sus ventas agropecuarias (productor 4): se trata de una mujer que vive sola, quien está bien integrada a la dinámica colectiva del grupo para vincularse al mercado cuencano. 
$\mathrm{Al}$ otro extremo nos encontramos con una familia (productor 5) cuyos ingresos provienen en $86 \%$ de los empleos urbanos. En este caso ya no vale hablar de hogar campesino porque las ventas de hortalizas, de frutas o de lácteos representan una parte marginal de la economía doméstica. Para la tercera familia (productor 3), las remesas representan el 50\% de los ingresos globales, lo que parece fundamental para la supervivencia del grupo cuando se nota el bajo nivel de actividad comercial. En los dos últimos casos observamos una situación intermediaria, con ingresos agropecuarios que representan el 37\% de la economía familiar (productor 2), y un caso exitoso, con el $75 \%$ de las fuentes monetarias familiares que vienen principalmente de las ventas de hortalizas y de frutas, de lácteos y de pequeños animales.

La conclusión parcial que podríamos hacer, después de analizar estas cifras, es que la inserción urbana de los campesinos de la parroquia Octavio Cordero Palacios es indudablemente un factor de mejoramiento de la economía familiar. Además, la orientación hacia un sistema productivo más intensivo en trabajo les permite llegar a su propia seguridad alimentaria. Sin hablar de revolución, podemos considerar que esta experiencia es muy interesante, pero la vía agroecológica sigue con debilidades.

\section{PROBLEMAS TÉCNICOS Y LIMITACIONES DE LAS REDES DE PRODUCTORES}

El auge de la agro-ecología en la provincia del Azuay no debe borrar las dificultades y los límites de la agricultura familiar en esta provincia. A la pregunta, ¿puede la agro-ecología constituir una vía de desarrollo rural en el contexto migratorio regional?; por el momento, no podemos responder de manera afirmativa, por varias razones:

- Primero, porque los productores que pueden vender en los mercados pertenecen a la periferia inmediata de la ciudad de Cuenca, lo cual significa que la mayoría de productores que viven en el resto de la provincia quedan afuera de la dinámica comercial. Entonces, no hablamos de redes regionales, sino de organizaciones de productores muy localizadas, cuya influencia geográfica es muy reducida todavía; 
- Segundo, porque las dos asociaciones no integran a los campesinos con pocas tierras; es decir, a los más pobres. Por eso sería necesario desarrollar formas de venta asociativas que permitieran integrar a un mayor número de productores aislados, como lo hemos visto a través del ejemplo del grupo Bajo Invernadero en la parroquia Octavio Cordero Palacios;

- Tercero, porque la producción agro-ecológica no es suficiente para aprovisionar la ciudad en el contexto actual. La entrada de más productores, que trabajen en grupos, aseguraría un aprovisionamiento más regular y más importante; mientras que, actualmente, los agroecológicos siguen siendo minoritarios en la ciudad de Cuenca con respecto a los miles de intermediarios presentes en los diferentes mercados. De hecho, la ampliación de la red asociativa podría ser la clave de la soberanía alimentaria regional.

- Cuatro, porque el seguimiento técnico sobre las normas agro-ecológicas no es fiable. Ni el municipio de Cuenca ni el CREA tienen la capacidad de controlar regularmente a todos los productores, lo cual provoca ciertas irregularidades en las producciones. En este contexto, la agro-ecología no es necesariamente una garantía de la calidad de los productos, sino una vía para asegurarse un espacio de venta. Entonces, sería importante promover algunos productores encargados de la formación y del control de la producción agro-ecológica regional, para que las redes sean administradas por los campesinos mismos.

El desarrollo de la agricultura comercial y el aprovisionamiento urbano no pueden limitarse a un grupo reducido de productores especializados en la producción de hortalizas. La diversificación agropecuaria tiene que ser el elemento central de la agricultura regional, para permitir a una mayoría de campesinos vincularse al mercado y vender sus productos. Además, la multiplicación de los puntos de ventas en toda la provincia debería ser prevista, para dar la oportunidad a los campesinos ubicados en las partes marginales de la región de acceder regularmente a un espacio 
de venta. Eso debería ser acompañado de un mejoramiento de las condiciones de transporte para aumentar la rentabilidad de la actividad campesina. En definitiva, el desarrollo de la agricultura comercial en la provincia del Azuay pasa por la integración de un número más importante de productores, por un fortalecimiento del apoyo institucional y por un ordenamiento del espacio regional para articular la capital, Cuenca, con su periferia rural.

\section{A MODO DE CONCLUSIÓN}

La emergencia de la agricultura comercial en la provincia del Azuay, y particularmente en la parroquia Octavio Cordero Palacios, aparece como un fenómeno interesante de las mutaciones actuales de la ruralidad andina. En efecto, hemos constatado que gracias a los apoyos de varias instituciones públicas, las pequeñas unidades de producción pueden ser eficientes para aprovisionar cotidianamente el mercado urbano de Cuenca, lo cual les permite sacar ingresos regulares. Así, la conexión entre la agricultura familiar y el medio urbano o, mejor dicho, el desarrollo de redes cortas de productores, podría ser a mediano plazo el factor clave del mantenimiento de las explotaciones familiares en esta región.

Sin embargo, no deben ignorarse las debilidades de la dinámica comercial regional. Por el momento, se nota que las redes de productores integran un número muy reducido de agricultores, mientras que la mayoría de las explotaciones de la provincia del Azuay, en particular las que se encuentran en las localidades más alejadas de la ciudad de Cuenca, no tienen acceso a los consumidores urbanos. Por eso la migración sigue siendo la principal alternativa para la sobrevivencia de muchas explotaciones. Por ello, los apoyos institucionales deberían ser más importantes en toda la provincia, tanto para el crecimiento y la diversificación de la producción regional, como para el mejoramiento de las economías familiares.

En el Ecuador, cuyo mercado agrícola ha sido desorganizado en las últimas décadas por la apertura liberal (Peltre-Wurtz, 2004), el desarrollo de redes de productores locales y el acercamiento entre las ciudades y las áreas rurales podrían ser los primeros pasos para actuar concretamente a favor del mantenimiento de la agricultura campesina. Eso permitiría el mejoramiento de las economías rurales y seguramente, la diversificación agrícola de las pequeñas 
unidades de producción, las cuales tendrían un rol mucho más importante para la seguridad alimentaria nacional. En definitiva, sería probablemente la estrategia más eficiente para luchar contra la migración y la proletarización de los campesinos.

\section{BIBLIOGRAFÍA}

Acosta, Alberto, Susana López y David Villamar, 2006, «Las remesas y su aporte para la economía ecuatoriana», en La migración ecuatoriana: transnacionalismo, redes e identidades, editado por Herrera, Gioconda et al., FLACSO, Quito, pp. 227-252.

Calas, Bernard, 1999, «Les paradoxes des rapports villes-campagnes à travers à travers l'analyse du ravitaillement kampalais», en Villes et campagnes dans les pays du sud : une géographie des relations, editado por Chaléard, Jean-Louis et al., Karthala, Paris, pp. 87-103.

Chaléard, Jean-Louis, 1996, Temps des villes, temps de vivres. L'essor du vivrier marchand en Côte d'Ivoire, Karthala, Paris.

Deler, Jean-Paul, 2007, Ecuador del espacio al estado nacional. IFEA/Universidad Simón Bolívar/Corporación Editora Nacional, Quito.

Dirección Nacional de Migraciones, Oficina de Estadísticas.

FLACSO, 2008, Ecuador: la migración en cifras, FLACSO/UNFPA, Quito.

Herrera, Gioconda, María Cristina Carrillo y Alicia Torres (eds.), 2006, La migración ecuatoriana: transnacionalismo, redes e identidades, FLACSO, Quito.

Herrera, Gioconda y Jacques Ramirez, (eds.), 2008, América Latina migrante: Estado, familia, identidades, FLACSO, Quito. 
IERSE, 2003, Registro de la información cartográfica de la cuenca del Paute, http://www.uazuay.edu.ec/geomatica/source/web/links/metadatos.html

INEC, 1982, 1990, 2001, Censos de Población.

Nieto, Marisol, 2005, «Las remesas, su influencia en la economía ecuatoriana y el dilema del desarrollo», en Emigración y política exterior en Ecuador, editado por Javier Ponce Leiva, FLACSO/Abya Yala, Quito, 197-221.

Peltre-Wurtz, Jacqueline, 2004, Luchar para comer: estrategias familiares para la alimentación en sectores populares, Abya Yala, Quito.

Poloni Simard, Jacques, 2006, El mosaico indígena. Movilidad, estratificación social y mestizaje en el corregimiento de Cuenca (Ecuador) del siglo XVI al XVIII, Abya Yala/IFEA, Quito.

Rebaï, Nasser, 2008, «Juncal de Cañar: la progresiva transformación territorial de una parroquia de la sierra ecuatoriana», Ecuador Debate, n. 75, CAAP, Quito, pp.107-116.

Tallet, Bernard, 1999, «Le maraîchage à Bobo-Dioulasso (Burkina Faso): un dynamisme agricole sous influence urbaine», en Villes et campagnes dans les pays du sud: une géographie des relations, editado por Chaléard, Jean-Louis et al., Karthala, Paris, pp. 49-59.

\section{NOTAS}

\footnotetext{
${ }^{1}$ El promedio de saldos migratorios de 1990 a 1999 era de 31,000 individuos, mientras que del 2000 al 2007 era de 106,000 (Dirección Nacional de Migración - DNM).

${ }^{2}$ El Austro corresponde a las tres provincias australes del Ecuador: Cañar, Azuay y Morona Santiago.

${ }^{3}$ Este nombre hace referencia a la acción del cura, quien animaba a los campesinos a construir invernaderos para producir y vender frutales. Ahora, constatamos que su influencia fue determinante para la agricultura local.
}

Fecha de recepción: $1^{\circ}$ de diciembre de 2010.

Fecha de aceptación: 19 de enero de 2012. 